\title{
Laboratorio virtual de software libre para bibliotecas
}

\author{
Por Patricia Russo-Gallo, Josep-Manel Rodríguez-Gairín y Andreu Sulé-Duesa
}

\begin{abstract}
Resumen: La elección de un programa de gestión de bibliotecas se ve afectada muchas veces por una serie de condiciones sociales, económicas y políticas que hacen que la elección no sea la más adecuada para las necesidades, características y funciones de la biblioteca. El software libre está siendo una de las soluciones más optadas, por sus libertades de copia, modificación y distribución, además de la libertad de licencias y las posibilidades de interoperación con otras aplicaciones. Esta nueva tendencia hacia el software libre en bibliotecas se refleja también en los estudios de biblioteconomía y documentación, en los que desde diferentes asignaturas se aportan conocimientos sobre programas de automatización, de gestión de repositorios, incluso del sistema operativo Linux/GNU, entre otros. Esta combinación entre las necesida-

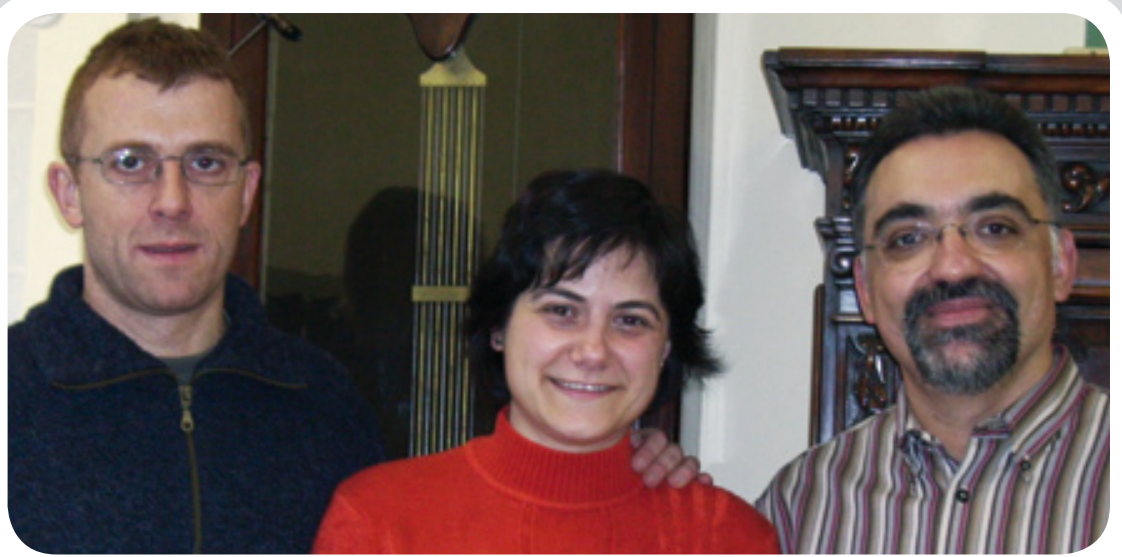

Andreu Sulé-Duesa es profesor de la Facultat de Biblioteconomia i Documentació de la Universitat de Barcelona en donde imparte asignaturas relacionadas con los sistemas de automatización. Profesor del curso virtual del Cobdc, Introducció al format Marc 21.

Patricia Russo-Gallo es coordinadora del Grupo de Trabajo de Software Libre para Profesionales de la Información (Cobdc). Trabaja como arquitecta de la información en una empresa de experiencia de marcas y diseño web (Abilbo) en Barcelona.

Josep-Manel Rodríguez-Gairín es profesor de la Facultat de Biblioteconomia i Documentació de la Universitat de Barcelona donde coordina también el Màster en gestió de continguts digitals, y es profesor del Master online de documentación digital del IDEC, Univ. Pompeu Fabra.

des de los centros y la tendencia al uso de software libre, es la que un grupo de profesores de la Facultat de Biblioteconomia i Documentació (Universitat de Barcelona) y estudiantes, miembros del Grup de Treball sobre Programari Lliure per als Professionals de la Informació (Cobdc), han querido aportar a la comunidad profesional, creando un laboratorio virtual para el uso de software libre de aplicación en bibliotecas.
\end{abstract}

Palabras clave: Software libre, Automatización de bibliotecas, Gestión documental, Tesauros, Depósitos digitales, Repositorios, Gestión de bibliografías, Gestión de recursos electrónicos, Educación virtual, Laboratorios virtuales.

\section{Title: Virtual laboratory of free software for libraries}

Abstract: The selection of a library management system is often affected by social, economic and political conditions that may result in an inappropriate choice for a library's needs, characteristics and functions. Free software, or freeware, is one of the more often selected solutions, given the freedom to copy, modify and distribute it in addition to free licensing and the possibilities for integration with other applications. This trend is reflected in librarianship curricula, in which automation software, repository management software, and even Linux/GNU, among others, are explained in a variety of courses. This combination of organizational needs and freeware trends led a group of professors from the Faculty of Library and Information Science (UB) and members of the Work Group on Free Software for Information Professionals (Cobdc) to create a virtual laboratory for the use of free software for library applications, as a contribution to the professional community.

Keywords: Free software, Freeware, Library automation, Document management, Thesaurus, Digital deposit, Repositories, Bibliography management, Electronic resources management, E-learning, Virtual lab.

Russo-Gallo, Patricia; Rodríguez-Gairín, Josep-Manel; Sulé-Duesa, Andreu. "Laboratorio virtual de software libre para bibliotecas". En: El profesional de la información, 2008, enero-febrero, v. 17, n. 1, pp. 71-77.

DOI: 10.3145/epi.2008.ene.08

\section{Introducción}

CON MOTIVO DEL FES-

INTERNET 2007, miembros del

Grup de treball sobre programari

lliure per als professionals de la informació del Col•legi Oficial de
Bibliotecaris-Documentalistes de Catalunya y de la Facultat de Biblioteconomia i Documentació de la Universitat de Barcelona decidimos crear un laboratorio virtual de software libre orientado a la au- tomatización de unidades de información (principalmente bibliotecas y centros de documentación).

http://www.fesinternet.net/

http://www.soft-libre.net/

http://www.ub.edu/biblio 


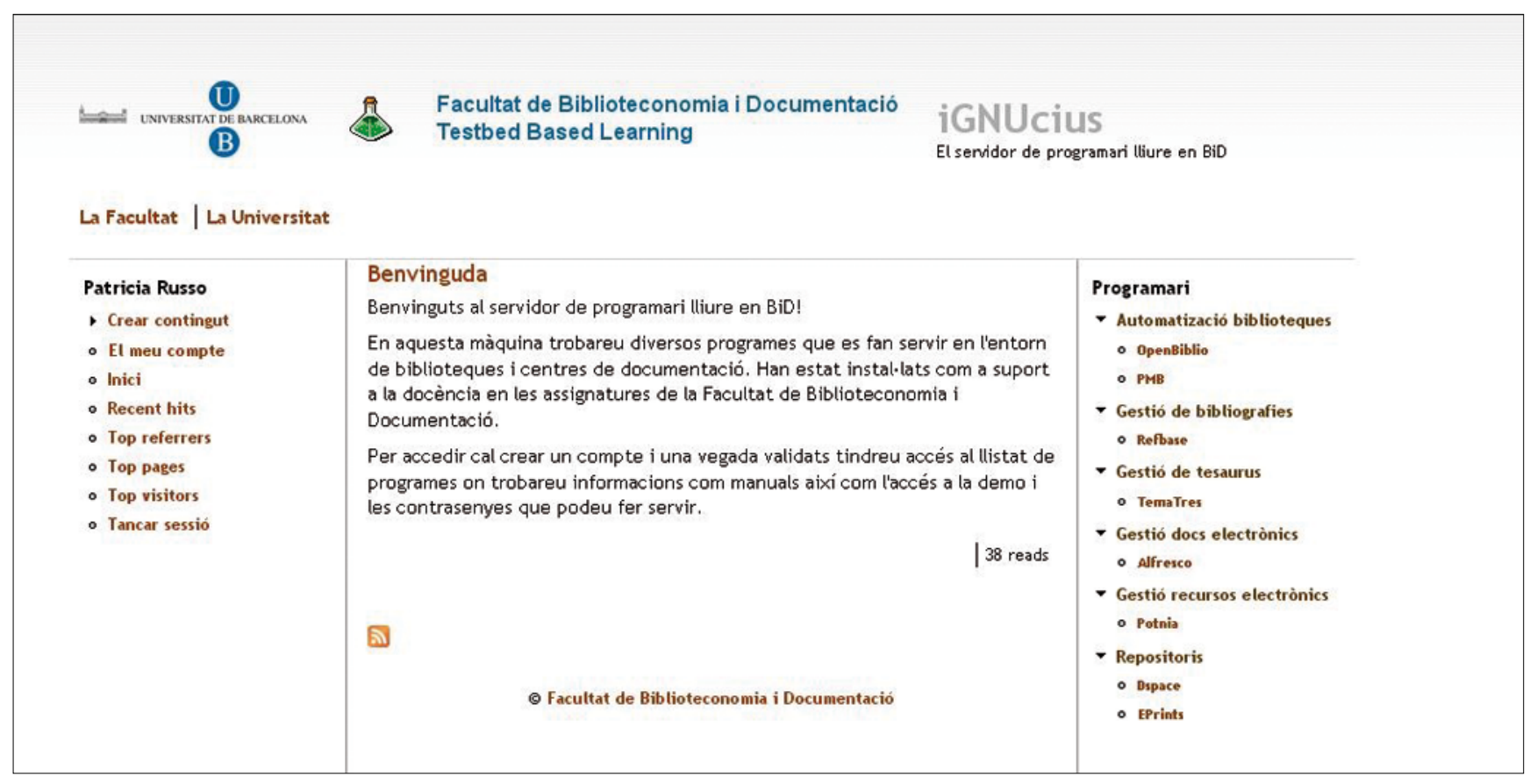

Web del laboratorio virtual

El objetivo era dar a conocer, tanto entre los alumnos de la Facultat como entre los profesionales del sector, diferentes soluciones gratuitas de automatización. Y como la mejor manera de conocer un programa es utilizándolo, además de las presentaciones de los diversos productos, centramos nuestros esfuerzos en la creación de un entorno virtual que permitiera a los interesados su testado directo, sin intermediarios. Durante los días que el laboratorio estuvo activo (del 15 de mayo al 8 de junio de 2007), los alumnos y los profesionales que accedieron a él pudieron gestionar los programas con todos los privilegios, administrando el sistema, creando cuentas de usuario, editando registros, creando estadísticas, importando registros, etc.

Para conseguir este objetivo se instalaron en un servidor (al que se le denominó iGNUcius, en honor del personaje que, a manera de broma, interpreta Richard Stallman) los programas siguientes:

- PMB y Openbiblio (automatización de bibliotecas).

- Potnia (gestión de recursos electrónicos).
- Alfresco (gestión de documentos electrónicos).

- Dspace y E-prints (gestión de repositorios).

- Tematres (gestión de tesauros).

- Refbase (gestión de bibliografías).

http://es.wikipedia.org/wiki/ San_Ignucio

\section{"Centramos nuestros esfuerzos en la creación de un entorno virtual que permitiera a los interesados experimentar en directo"}

Además del acceso a todas sus funciones, en la misma interfaz web se ofrecía una breve descripción de las principales características de cada producto, acceso a su web oficial y a los manuales de instalación y uso.

\section{Programas expuestos}

A continuación se describen las principales funciones y características de todos ellos.

\section{PMB}

Es un sistema de automatización de bibliotecas creado en Francia en 2002 bajo licencia CeCILL, una licencia de software libre del gobierno francés que recoge las mismas condiciones y libertades de la que $G P L$.

\section{http://www.sigb.net}

Está destinado a bibliotecas medianas y grandes, permitiendo también la gestión de una red de bibliotecas integradas en un catálogo colectivo. El formato de catalogación que utiliza es Unimarc pero incorpora un sistema de conversión e importación de formatos Usmarc y xml entre otros.

Lo podemos encontrar traducido a varios idiomas, entre ellos francés, inglés, español y catalán. Está disponible la versión 3 desde septiembre de 2006.

a. Requisitos técnicos mínimos de la máquina y programas necesarios para la instalación: 

GHz.

- Procesador: Intel Pentium 1

- Memoria: 256 MB.

- Sistema operativo: GNU-Linux, Windows 2000/XP, MacOS, etc.

- Lenguaje de programación: PHP v. 4.2. 4.0 .

- Base de datos: $M y S Q L$ v.

- Navegador: Mozilla Firefox, IE 6.0, Netscape 7, etc.

b. Características.

- Gestión de autoridades (por autores, editores, colecciones). terias.

- Gestión de un tesauro de ma-

- Uso del protocolo Z39.50.

- Automatización del servicio de DSI. serie.

- Control de publicaciones en

- Gestión de vaciado de revistas.

- Sistema de copias de seguridad.

c. Módulos.

- Administración del sistema, desde donde se puede parametrizar toda la configuración general, gestionar a los usuarios, realizar las copias de seguridad, etc.

- Catalogación, para monografías, publicaciones en serie, captura de registros desde el catálogo Z39.50, creación de listas de registros (de novedades, búsquedas guardadas, etc.).

- Préstamo.

- Autoridades para autores, editores, colecciones y clasificación. Incluye la creación de un tesauro para materias.

- DSI.

- Opac.

\section{Openbiblio}

Es un sistema integrado de gestión de bibliotecas. Creado en 2002 en inglés y bajo licencia $G N U-G P L$.
Está disponible en diversas lenguas, entre ellas español y catalán.

\section{http://obiblio.sourceforge.net}

Está destinado a pequeñas bibliotecas, sobre todo escolares. El formato de catalogación es Unimarc pero permite la importación de registros en otros compatibles. Está disponible la versión 0.6.0 desde febrero de 2007.

a. Requisitos.

- Sistema operativo: GNU-Linux, Windows 2000/XP.

- Lenguaje de programación: PHP v. 4.2 . 4.0.

- Base de datos: MySQL v.

- Navegador: Mozilla Firefox, IE 6.0, Netscape 7, etc.

b. Características.

- Avisos a los usuarios a través de mensajes prediseñados en el sistema.

- Control de multas por retraso en la devolución de documentos.

- Diversos modelos de estadísticas de uso de la biblioteca y el material.

c. Módulos.

- Préstamo. Administración de datos de los usuarios, sus préstamos, devoluciones, reservas y multas.

- Catalogación. Control de registros bibliográficos y las respectivas copias.

- Administración. Configuración y gestión del sistema, incluidas las autorizaciones del personal administrador del sistema.

- Informes. Gestión de estadísticas, informes y mensajes a los usuarios.

- Opac.

\section{Potnia}

Es un programa para la creación de directorios temáticos pensado para la gestión de recursos científicos, revistas, publicaciones, etc. Creado en 2003 bajo un proyecto de la Dirección General de Universidades del Ministerio de Educación, Cultura y Deportes (España). Se rige por la Mozilla public license 1.1 (MPL 1.1). Originalmente en español, no se conocen otras traducciones.

\section{http://potnia.sourceforge.net}

La estructura y la descripción de los registros siguen un estándar definido por el conjunto de metadatos Dublin Core. Se encuentra disponible la versión 1.0 desde octubre de 2003.

a. Requisitos.

- Servidor: Apache.

- Sistema operativo: GNU-Linux, Windows 2000/XP.

- Lenguaje de programación: PHP v. 4.2 4.0 .

- Base de datos: $M y S Q L$ v.

- Navegador: Mozilla Firefox, IE 6.0, etc.

b. Funciones.

- Búsqueda de registros. Además de la consulta básica también se incluye una avanzada que permite concretar los campos donde se quiere buscar (título, palabra clave, descripción o tema) y la combinación de éstos a través de los operadores booleanos and, or y not.

- Administración del sistema. Permite añadir, modificar o borrar registros de la base de datos y también gestionar la lista de temas.

- Página de inicio. Ofrece la lista de temas de los registros de la base de datos; una vez dentro de uno de los temas se presenta la lista de los registros categorizados bajo el mismo, desde donde se podrá acceder al detalle.

\section{Alfresco}

Se define como un gestor de contenidos empresariales (enterprise content management, ECM) y entre sus aplicaciones está la gestión de documentos. Creado en 
2005 por un equipo de miembros de Documentum e Interwoven. Originalmente en inglés, no se conoce una versión oficial en español.

\section{http://www.alfresco.com}

Es un software de código abierto. El acceso al código fuente permite un alto nivel de calidad en el desarrollo y evolución del sistema. El beneficio comercial se halla en el empaquetado junto con los manuales, formación y mantenimiento del programa.

Dispone de un espacio dedicado a las transformaciones que la comunidad de usuarios realiza sobre el programa, las cuales están bajo una licencia Alfresco public license. Disponible la versión 2.1 desde junio de 2007.

\section{http://forge.alfresco.com/}

a. Requisitos.

- Servidor: Apache Tomcat.

- Sistema operativo: GNU-Linux, Windows 2000/XP.

- Lenguaje de programación: PHP v. 4.2 . 4.0.

- Base de datos: $M y S Q L$ v.

- Navegador: Mozilla Firefox, IE 6.0, etc.

\section{b. Características.}

- Depósito de documentos electrónicos en su formato original (doc, xls, tif, html, jpg, pdf, vídeo, audio, cad, etc.).

- Visualizadores de documentos.

- Agrupaciones por carpetas y cuadro de clasificación.

- Sistema de recuperación de la información, búsqueda básica y avanzada, busca ficheros, carpetas y contenidos.

- Gestión y control de flujos de trabajo.

- Gestión de usuarios y niveles de acceso.

- Histórico de versiones. c. Funciones.

- Espacio común desde donde clasificar la documentación por carpetas.

- Permite añadir documentos de la red local o crearlos nuevos desde el mismo sistema (en formato html, txt o xml).

- Gestiona reglas de contenidos con las que transformar formatos de forma automática, crear flujos de trabajo, etc.

- Espacios de foros para las carpetas o documentos concretos.

- Edición y modificación de los metadatos de cada documento, desde donde también ver el histórico de las versiones creadas.

- Creación de copias de trabajo.

- Gestión de los perfiles de usuarios del sistema y sus privilegios y autorizaciones.

\section{DSpace}

Es un sistema de gestión de depósitos institucionales (repositorios) creado por la Biblioteca del MIT (Massachusetts Institute of Technology) y Hewlett-Packard en 2002. Se distribuye bajo BSD Open source licenses (Berkeley Software Distribution), que se encuentra dentro de la familia de las consideradas como software libre, pero con algunas restricciones en comparación con la licencia GNU-GPL. Permite el uso y modificación del código fuente y el uso comercial del producto.

\section{http://www.dspace.org}

Es posible la gestión de diferentes tipos de materiales (preprints, informes técnicos, imágenes, vídeos, etc.) y contribuye a la preservación digital de la producción científica de una organización. Lo podemos encontrar traducido a diversos idiomas, entre ellos el español, catalán y gallego.

Como sistema de preservación de material digital, a todos los re- gistros se les asigna un identificador persistente. Dspace trabaja, como Handles, con el Protocol for Metadata Harvesting (PMH) de la Open Archive Initiative. Está disponible la versión 1.4.2 desde mayo de 2007.

a. Requisitos.

- Servidor: Apache Tomcat.

- Sistema operativo: Unix, GNU-Linux, Solaris. Java.

- Lenguaje de programación:

- Base de datos: postgreSQL.

- Navegador: Mozilla Firefox, IE 6.0, etc.

b. Características.

- Soporta cualquier tipo de material: documentos, tesis, imágenes, audio, vídeo, etc.

- Los registros quedan guardados en formato Dublin Core.

- Puede interoperar con otros sistemas de la organización.

- Todos los registros tienen un identificador persistente.

- Permite la migración de formatos hacia $I F F, S G M L$, xml, $A I F F$, pdf, y, aunque no se asegura, hacia otros propietarios como Microsoft Word, PowerPoint, Lotus 1-2-3 o WordPerfect.

\section{E-Prints}

Es un sistema de creación de repositorios institucionales de la producción científica, tesis, informes, etc. Creado por la University of Southampton (Reino Unido) en 2000 y distribuido con licencia GNU-GPL. Originalmente en inglés, podemos encontrar alguna versión traducida al español.

\section{http://www.eprints.org/}

Utiliza formato de metadatos xml y Dublin Core basado en la $O A I-P M H$, lo que hace que pueda soportar diferentes tipos de documentos y formatos de datos para su preservación y acceso abierto a los contenidos y su conocimiento. Está 
disponible la versión 3 desde enero de 2007.

a. Requisitos.

- Procesador: $1 \mathrm{GHz}$.

- Memoria: 512 MB.

- Servidor: Apache.

- Sistema operativo: Unix, GNU-Linux. PERL.

- Lenguaje de programación:

- Base de datos: $M y S Q L$.

- Navegador: Mozilla Firefox, IE 6.0, etc.

b. Características.

- Formato de metadatos Dublin Core.

- Repositorio de todo tipo de material: texto, imagen, etc.

- Sindicación de contenidos.

- Incluye un gestor de bibliografías.

- Búsqueda básica y avanzada.

\section{Tematres}

Es una aplicación web para la gestión de tesauros creada en Argentina en 2004 bajo licencia GPL y originalmente en español. También está pensada para la creación de estructuras de navegación web, directorios de bibliotecas digitales o control de lenguajes documentales. Disponible la versión 0.9.5 desde mayo de 2007. tres

http://www.r020.com.ar/tema-

a. Requisitos.

- Servidor: Apache.

- Sistema operativo: GNU-Linux, Windows 2000/XP.

- Lenguaje de programación: PHP v. 4.2.

- Base de datos: $M y S Q L$ v. 4.0.

- Navegador: Mozilla Firefox, IE 6.0, etc.

b. Características.

- Relación entre los términos por: equivalencia, jerarquía y/o asociación.
- Cantidad ilimitada de términos relacionados, de niveles jerárquicos y de términos no preferentes.

- Múltiples notas de alcance, históricas y bibliográficas por término.

- Control de términos repetidos.

- Navegación sistemática y alfabética.

- Formatos de exportación en xml, Dublin Core, RDF SKOSCore, Zthes, etc.

- Buscador general.

\section{RefBase}

Es un programa de gestión de bibliografías y citas bibliográfica que funciona bajo interfaz web multiusuario. Creado en Alemania en 2002 bajo licencia $G N U-G P L$ y originalmente en inglés, no se conoce ninguna versión en otros idiomas.

\section{http://refbase.sourceforge.net/}

Está pensado para crear colecciones de referencias bibliográficas importadas de diferentes bases de datos académicas y extraerlas en formato de cita para la bibliografía de textos. Está disponible la versión 0.9.0 desde octubre de 2006 .

a. Requisitos.

- Servidor: Apache.

- Sistema operativo: GNU-Linux, Windows 2000/XP.

- Lenguaje de programación: PHP v. 4.2. 4.0.

- Base de datos: $M y S Q L$ v.

- Navegador: Mozilla Firefox, IE 6.0, etc.

b. Características.

- Búsqueda básica o avanzada.

- Sindicación de contenidos.

- Formato de las citas: html, RTF, pdf, LaTeX.

- Formatos de importación: BibTeX, Endnote, RIS, RefWorks,
PubMed, ISI Web of Science, CSA Illumina, Copac, MODS XML.

- Formato de exportación: BibTeX, Endnote, RIS, MODS XML, $\mathrm{Xml}$ de la Library of Congress $\mathrm{y}$ OpenDocument.

\section{Aspectos técnicos de la puesta en marcha del laboratorio}

Fue necesario planificar previamente los requisitos de hardware y software base necesarios para soportar las aplicaciones escogidas. La Facultat de Biblioteconomia $i$ Documentació facilitó la adquisición de un servidor exclusivo $D E L L$ PowerEdge de doble procesador a 3.0 GHz, $2 \mathrm{MB}$ de memoria RAM y dos discos duros de 80 GB.

El sistema operativo escogido fue Linux en su distribución Debian GNU/Linux 4.0 etch sobre el que se instalaron las aplicaciones básicas requeridas por las distintas aplicaciones: Perl y PHP como lenguajes de programación, el entorno Java de desarrollo, Apache 2 como servidor web, TomCat 5.5 como servidor de aplicaciones, y $M y S Q L$ 5.0.2 y PostgreSQL 8.0 como sistemas de gestión de bases de datos. Se utilizó como antivirus ClamAV y se implantó un sistema de copias de seguridad.

Se escogió también un sistema de gestión de contenidos para la navegación por el laboratorio que permitiera registro de usuarios y control estadístico sobre el que se elaboraron las fichas descriptivas de cada uno de los programas. El CMS escogido fue Drupal.

A nivel técnico podemos diferenciar dos tipos básicos de aplicaciones:

- Las que se distribuyen en forma de scripts para ser utilizadas bajo un entorno Apache-PHP-MyS$Q L$. Su instalación normalmente sólo implica descomprimir los archivos en un directorio web y ejecutar un fichero de configuración 
desde el propio navegador que crea la base de datos y tablas necesarias ajustándose a los parámetros. Este tipo de aplicaciones suelen ser las más sencillas de instalar y en este grupo tenemos PMB, OpenBiblio, TemaTres y Potnia.

- Las basadas en entorno Java que requieren un servidor de aplicaciones como TomCat o basadas en PERL. Se distribuyen en código fuente y deben ser compiladas y enlazadas en el propio servidor. El proceso no resulta sencillo y puede requerir la instalación de módulos de PERL adicionales u otros complementos. En este grupo tenemos Alfresco, DSpace y Eprints.

Compaginar en una misma máquina todas las aplicaciones fue un proceso francamente complicado y tanto la experiencia práctica como los conocimientos compartidos con otros usuarios nos permiten hacer una serie de reflexiones.

Los procesos de instalación requieren unos conocimientos previos informáticos y no están al alcance del público en general, en especial comparando con versiones Windows de la misma aplicación (Alfresco, Eprints).

No resulta nada fácil ajustar compatibilidades entre ellas. Por ejemplo las aplicaciones basadas en PHP-MySQL pueden requerir distintos ajustes de configuración de PHP (register globals) o versiones específicas de $P H P$, ya sea porque empleen funciones nuevas, solamente presentes a partir de $\mathrm{PHP5}$, o bien otras obsoletas no compatibles con éste y que requieren por tanto PHP4. Este mismo problema se nos planteaba entre Alfresco (que se distribuye con una versión de TomCat concreta) y DSpace que requiere una preinstalación del mismo.

En el caso de DSpace, que podía utilizar distintos gestores de bases de datos como MySQL, Oracle o PostgreSQL, aunque parecía lógico emplear $M y S Q L$ (ya que era el que estábamos utilizando en el resto) los comentarios en listas de distribución y la complejidad de ajustes nos decantaron a usar PostgreSQL que es el gestor propuesto por defecto por los diseñadores de la aplicación.

La tabla 1 recoge las aplicaciones básicas empleadas por cada uno de los programas seleccionados.

Una vez superada la fase de instalación se requiere otra de pruebas pues hay que hacer ajustes técnicos algo complejos que no siempre están indicados en el manual y a los que se llega a través de las consultas a foros y listas de distribución.

La mayoría de estos programas demandan un seguimiento importante de las actualizaciones ya que pueden presentar fallos de seguridad. Sirva como ejemplo comentar que durante la exposición el servidor fue atacado y se le instaló software de phishing a través de un agujero de seguridad de $P M B$, detectado y corregido en una versión posterior a la de la instalación.

En este mismo aspecto es importante comentar que en muchos casos las personalizaciones y posibilidades de adaptación y traducción que ofrecen estos programas pueden condicionar las actualizaciones futuras y requerir un esfuerzo adicional importante para adecuar los desarrollos y completar las traducciones en las nuevas versiones del programa.

\section{Conclusiones}

Esta actividad, que empezó sólo para probar y promocionar software libre, ha permitido que la Facultad creara un espacio de aprendizaje tanto para profesores como para estudiantes, ofreciendo material de prácticas docentes para diferentes asignaturas. La posibilidad de interactuar directamente con los programas, así como la facilidad de acceso a todas sus funciones (evitando así el a veces farragoso proceso de instalación en una unidad local), ha permitido a los participantes en este proyecto conocer de primera mano diferentes soluciones gratuitas, comprobar su utilidad ante problemas y necesidades concretas, y resolver dudas e inquietudes de manera individual. Más allá de las simples demos o charlas de difusión, con este laboratorio virtual hemos pretendido difundir este tipo de programas de una manera prác-

\begin{tabular}{|l|c|c|c|c|c|c|c|c|}
\hline & OpenBiblio & PMB & RefBase & TemaTres & Alfresco & Potnia & Eprints & Dspace \\
\hline Apache & $\mathrm{X}$ & $\mathrm{X}$ & $\mathrm{X}$ & $\mathrm{X}$ & & $\mathrm{X}$ & $\mathrm{X}$ & \\
\hline Tomcat & & & & & $\mathrm{X}$ & & $\mathrm{X}$ \\
\hline PHP & $\mathrm{X}$ & $\mathrm{X}$ & $\mathrm{X}$ & $\mathrm{X}$ & & $\mathrm{X}$ & Opt \\
\hline Perl & & & & & & & $\mathrm{X}$ & \\
\hline Java & & & & & $\mathrm{X}$ & & $\mathrm{X}$ \\
\hline MySQL & $\mathrm{X}$ & $\mathrm{X}$ & $\mathrm{X}$ & $\mathrm{X}$ & $\mathrm{Opt}$ & $\mathrm{X}$ & $\mathrm{X}$ & Opt \\
\hline PostgresQL & & & & & & & $\mathrm{X}$ \\
\hline
\end{tabular}


tica, de manera que fuera el propio interesado el que fuera descubriendo las posibilidades de cada programa.

\section{Bibliografía}

Arano, Silvia. "Los tesaurus y las ontologías en la biblioteconomía y la documentación". En: $\mathrm{Hi}$ pertext.net, 2005, n. 3. Consultado en: 29-09-07. http://www.hipertext.net

Crow, Raym. "Institutional repository software options: questions and answers with the experts, with representatives from ARNO, CDSware, DSpace, Fedora, GNU Eprints, i-Tor, MPG eDoc and MyCoRe". En: CERN workshop on innova tions in scholarly communication: implementing the benefits of OAI (OAI3), 2004.

Russo-Gallo, Patricia. "PMB: programari lliure d'automatització de biblioteques". En: BiD. textos universitaris de biblioteconomia $i$ documentació, 2006, desembre, n. 17. Consultado en: 24-09-07.

http://www2.ub.edu/bid/consulta_articulos. php?fichero $=17$ russo.htm

Serrano-Muñoz, Jordi; Prats-Prat, Jordi "Repertorios abiertos: el libre acceso a contenidos". En: Revista de universidad y sociedad del conocimiento, 2005, v. 2, n. 2.

Tramullas, Jesús. "Herramientas de software libre para la gestión de contenidos". En: Hipertext. net, 2005, n. 3. Consultado en: 29-09-07. http://www.hipertext.net
Patricia Russo-Gallo, Abilbo, Barcelona.

prusso@uoc.edu

Josep-Manel Rodríguez-Gairín, Facultat de Biblioteconomia i Documentació, Universidad de Barcelona.

rodriguez.gairin@ub.edu

Andreu Sulé-Duesa, Facultat de Biblioteconomia i Documentació, Universidad de Barcelona. sule@ub.edu

\section{Bienvenidos a la universidad personal}

Na pueda perder tiempa can desplazamientos. Perc los 30 seyuindos que lar do en lleyar al ordenadar me parecen razohables.

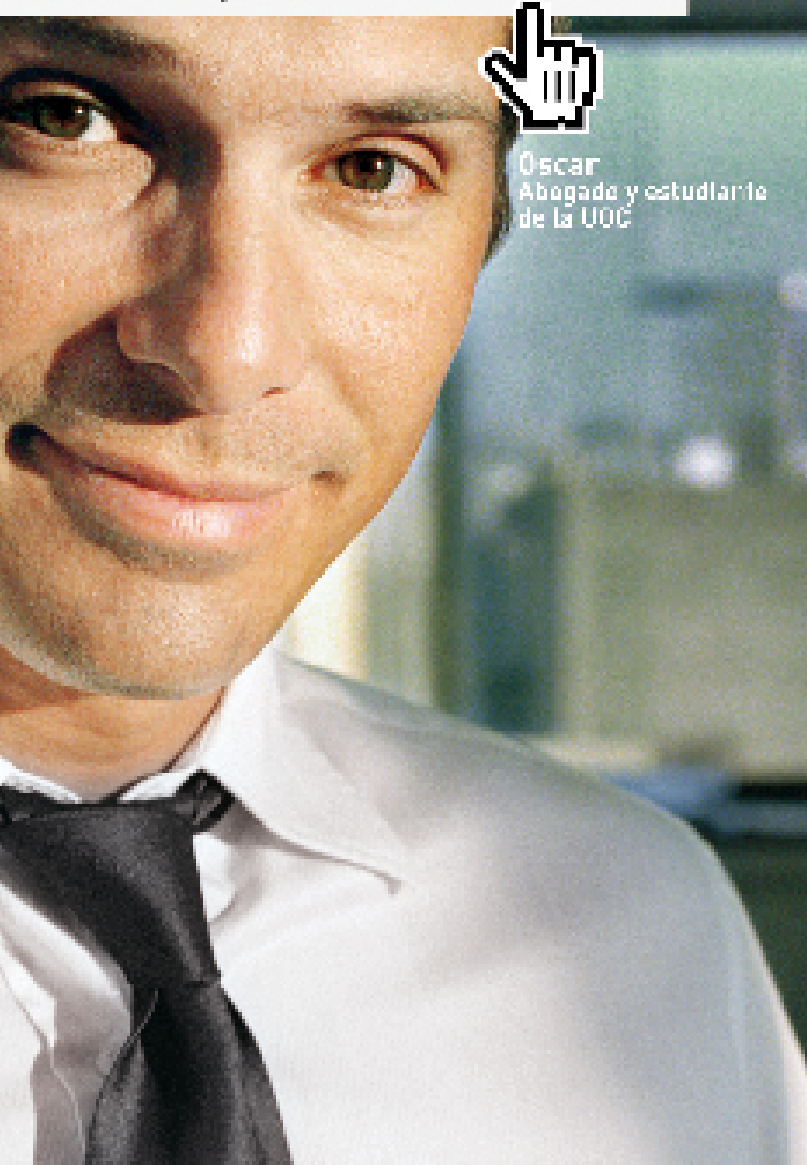

\section{ESTUDIOS DE CIENCIAS DE LA INFORMACIÓN Y DE LA COMUNICACIÓN}

Formamas a lce profesianales de la gnetiain de la infarmacián y la comuriagrián.

\section{Licenciaturas de 2: ciclo:}

- Liescislura de Dosumenlysiōn

- Lesclatura da Publkidad y Rolacierea Fíblesa

- Licscrislura de Cemuniacikin sudiovizual

\section{Pregramas de Posgrado:}

- Mijitar de Editicín

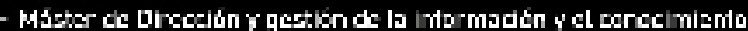

un las vrganizaticras

Peagrads de Psriedisma dig tal UDE - El Perífdien

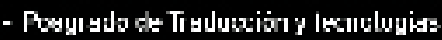

Inkicié: 1ebrero 2ulus

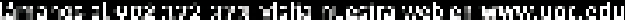

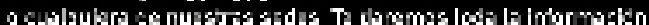

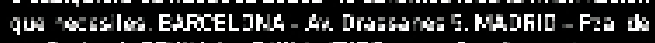

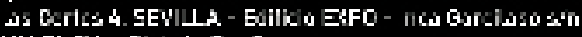

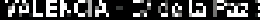

